\title{
ELLIPTIC SPACES
}

\author{
YVES FÉLIX, STEPHEN HALPERIN, AND JEAN-CLAUDE THOMAS
}

\begin{abstract}
A simply connected CW complex of finite type is elliptic if it has finite L.S.-category and if its loop space homology has polynomial growth.
\end{abstract}

Theorem A. If $X$ is elliptic it has the homotopy type of a finite Poincaré complex.

Theorem B. If $X$ is elliptic then $H_{*}(\Omega X)$ is a left noetherian ring.

Let $\Omega X$ denote the loop space of a nontrivial simply connected CW complex $X$. One of the first applications of the Serre spectral sequence was the theorem [15] that $X$ and $\Omega X$ could not both have homology concentrated in finitely many degrees. If we weaken these conditions slightly, and require that $X$ have finite Lusternik-Schnirelmann category and that the homology of $\Omega X$ grow at most polynomially, we obtain the class of elliptic spaces, which we introduce here (the precise definition follows).

In the rough analogy between spaces $X$ and local rings $R$ with residue field $\mathbf{k}$, the homology of $\Omega X$ corresponds to $\operatorname{Tor}_{*}^{R}(k, k)$. Since polynomial growth in the latter characterizes complete intersections [9], we may think of elliptic spaces as analogues of local complete intersections.

As we see with Theorems A and B to follow, ellipticity is a very severe restriction on a topological space. It is therefore somewhat remarkable that many of the spaces which play an important role in geometry are, in fact, elliptic. For example, simply connected Lie

Received by the editors September 26, 1990 and, in revised form, December 19, 1990.

1980 Mathematics Subject Classification (1985 Revision). Primary 55P35, 57P10, 57T25.

Key words and phrases. Elliptic, loop space homology, depth, L.S.-category, Poincaré complex.

The second author's research was supported in part by an NSERC operating grant.

The third author was supported by U.R.A. au C.N.R.S. 751.

This research partially supported by a NATO travel grant held by the three authors. 
groups and homogeneous spaces (in particular, spheres) are elliptic. More generally, simply connected finite $H$-spaces are elliptic, and indeed so are simply connected finite complexes whose loop space homology algebra is commutative [4], and the $H$-space analogues of homogeneous spaces [5]. Fibrations built out of elliptic spaces are elliptic; this then includes sphere bundles and principal bundles over elliptic manifolds.

In Riemannian geometry, the simply connected Dupin hypersurfaces in $S^{n}$ are elliptic [7]. Moreover all known examples of simply connected positively curved manifolds are elliptic, and a conjecture of Bott asserts that they all should be. Finally, the connected sums $\mathbf{C} P^{n} \# \mathbf{C} P^{n}, \mathbf{H} P^{n} \# \mathbf{H} P^{n}$ and $\mathbf{K} P^{2} \# \mathbf{K} P^{2}$ are elliptic, but these are essentially the only elliptic connected sums.

We come now to the precise definition, and to Theorems A and $\mathrm{B}$, which are the main object of this note. In the following, $H_{*}(-)$ denotes singular homology with integral coefficients, $p$ is an (arbitrary) prime, and $R$ is always a subring of $\mathbf{Q}$.

Definition. A simply connected CW complex, $X$, is called elliptic (or Z-elliptic) if it satisfies the following conditions:

(i) $X$ has finite Lusternik-Schnirelmann category.

(ii) Each $H_{i}(X)$ is finitely generated.

(iii) For each $\mathbf{k}=\mathbf{Z}_{p}$ or $\mathbf{Q}$ there is an integer $N=N(\mathbf{k})$ and a constant $C=C(\mathbf{k})$ such that

$$
\operatorname{dim} H_{r}(\Omega X ; \mathbf{k}) \leq C r^{N}, \quad r=1,2, \ldots .
$$

More generally, for any $R \subset \mathbf{Q}, X$ is $R$-elliptic if the localization $X_{R}$ satisfies (i) and (iii) and if each $H_{i}\left(X_{R}\right)$ is a finitely generated $R$-module.

In the presence of conditions (i) and (ii) the loop space homology tends to be relatively small (the elliptic case) or very large. In the rational case, which has been considered in some detail (eg. $[10,8,11])$, this is precise: either $X$ is a rationally elliptic or else its rational loop space homology grows exponentially [2].

In this note we prove the following two theorems:

Theorem A. If $X$ is a simply connected and R-elliptic CW complex, then $X_{R}$ satisfies Poincaré duality in the following sense: for some $n$

$$
H_{i}\left(X_{R}\right)=0, \quad i>n, \quad H_{n}\left(X_{R}\right) \cong R
$$


and if $\omega \in H_{n}\left(X_{R}\right)$ is a generator, then

$$
\cap \omega: H^{*}\left(X_{R}\right) \stackrel{\cong}{\rightrightarrows} H_{*}\left(X_{R}\right) \text {. }
$$

is an isomorphism. In particular, elliptic spaces have the homotopy type of finite Poincaré complexes.

Recall that $H_{*}(\Omega X)$ is an algebra with multiplication induced from composition of loops.

Theorem B. If $X$ is simply connected and R-elliptic CW complex then $H_{*}\left(\Omega X_{R}\right)$ is left (and right) noetherian. In particular, it is a finitely generated $R$-algebra.

The proofs of Theorems A and B, which follow, depend on the following ingredients, some of which we announce here with details to appear elsewhere.

Theorem 1 [3, Theorem B]. Suppose $X$ is 1-connected, cat $X \leq$ $\infty$ and for some constants $C$ and $N$, $\operatorname{dim} H_{r}(\Omega X ; \mathbf{k}) \leq C r^{N}$, $r=1,2, \ldots$, where $\mathbf{k}$ is a field. Then $H^{*}(X ; \mathbf{k})$ is a finite dimensional Poincaré duality algebra.

Theorem 2 [12, Theorem A]. Let $X$ be a simply connected $f$ nite $\mathrm{CW}$ complex. Suppose $1 / p \in R$ for $p \leq \operatorname{dim} X$ and that $H_{*}(\Omega X ; R)$ has no torsion. Then $H_{*}(\Omega X ; R)=U L$, where $L$ is the primitive sub Lie algebra.

We note that Theorem 2, which generalises the Milnor-Moore theorem [14], depends heavily on the work of Anick [1].

Theorem 3 ([13]). If $X$ is a rationally elliptic simply connected finite $\mathrm{CW}$ complex, then $H_{*}(\Omega X)$ has p-torsion for only finitely many primes $p$.

Theorem 4 [3, 4]. If $X$ is a finite simply connected CW complex such that for some $C, N: \operatorname{dim} H_{r}\left(\Omega X ; \mathbf{Z}_{p}\right) \leq r^{N}, r=1,2, \ldots$, then $H_{*}\left(\Omega X ; \mathbf{Z}_{p}\right)$ is a finite module over a central polynomial subalgebra $\mathbf{Z}_{p}\left[y_{1}, \ldots, y_{l}\right]$.

Theorem 5 [6]. If $X$ is $\mathbf{Z}_{(p)}$-elliptic then for some $m, p^{m}$ annihilates all the torsion in $H_{*}\left(\boldsymbol{\Omega} X ; \mathbf{Z}_{(p)}\right)$.

Proof of Theorem A. If $X$ is $R$-elliptic Theorem 1 shows that $H^{*}(X ; \mathbf{k})$ is a finite dimensional Poincaré duality algebra for any residue field $\mathbf{k}$ of $R$. The top dimension classes of these algebras are integral, because of Poincare duality, and so for some $n, H_{i}\left(X_{R}\right)=0, i>n$ and $H_{n}\left(X_{R}\right) \cong R$. 
Let $\omega \in H_{n}\left(X_{R}\right)$ be a generator. Then $\bigcap \omega: H^{*}\left(X_{R} ; \mathbf{k}\right) \stackrel{\cong}{\rightrightarrows}$ $H_{*}\left(X_{R} ; \mathbf{k}\right)$ for each residue field of $R$. Hence, by the universal coefficient theorem, $\cap \omega: H^{*}\left(X_{R}\right) \otimes \mathbf{k} \rightarrow H_{*}\left(X_{R}\right) \otimes \mathbf{k}$ is injective, and hence an isomorphism. It follows that $\bigcap \omega: H^{*}\left(X_{R}\right) \rightarrow H_{*}\left(X_{R}\right)$ is surjective with torsion kernel. In particular it restricts to a surjection between the torsion subgroups, which have the same number of elements, and so it is an isomorphism there. Hence $\bigcap \omega$ is an isomorphism.

Proof of Theorem B. By Theorem A, $X$ has the $R$-homotopy type of a finite complex and so we may assume it is one. Let $R^{\prime} \subset \mathbf{Q}$ be the subring obtained from $R$ by adjoining $1 / p$ to $R$ whenever $p<\operatorname{dim} X$ or $H_{*}(\Omega X ; R)$ has $p$-torsion.

Then Theorem 2 asserts that $H_{*}\left(\Omega X ; R^{\prime}\right)=U L$, where $L$ is the primitive sub Lie algebra. But since $X$ is necessarily rationally elliptic $L \otimes \mathbf{Q}$ is finite dimensional [4]. Thus $L$ is a free finitely generated $R^{\prime}$-module, and $H_{*}\left(\Omega X ; R^{\prime}\right)$ is left noetherian, by a standard mild generalization of the Hilbert Basis theorem.

On the other hand, the McGibbon-Wilkerson theorem (Theorem 3) implies that $R^{\prime}=R\left(1 / p_{1}, 1 / p_{2}, \ldots, 1 / p_{s}\right)$. Another standard argument shows that a graded $R$-algebra $A$ is left noetherian if each $A_{i}$ is a finitely generated $R$-module, and if for some $p$, $A \otimes_{R} R(1 / p)$ and $A \otimes_{R} \mathrm{Z}_{p}$ are both left noetherian. Thus a simple induction reduces us to showing $H_{*}(\Omega X) \otimes \mathbf{Z}_{p}$ is left noetherian for each noninvertible $p \in R$.

Use Theorem 4 to write $H_{*}\left(\Omega X ; \mathbf{Z}_{p}\right)$ as a finite module over $\mathbf{Z}_{p}\left[y_{1}, \ldots, y_{s}\right]$. Since the Bockstein differentials are derivations, $x_{i}=y_{i}^{p^{m}}$ survives to the $(m+1)$ st term of the Bockstein spectral sequence, and so Theorem 5 implies that $x_{i} \in H_{*}(\Omega X) \otimes$ $\mathbf{Z}_{p}$. Thus $H_{*}(\Omega X) \otimes \mathbf{Z}_{p} \subset H_{*}\left(\Omega X ; \mathbf{Z}_{p}\right)$ is a finite module over $\mathbf{Z}_{p}\left[x_{1}, \ldots, x_{l}\right]$ and so $H_{*}(\Omega X) \otimes \mathbf{Z}_{p}$ is left noetherian.

\section{REFERENCES}

1. D. Anick, Hopf algebras up to homotopy, J. Amer. Math. Soc. 2 (1989), 417-453.

2. Y. Félix, S. Halperin, and J.-C. Thomas, The homotopy Lie algebra for finite complexes, Publ. Math. Inst. Hautes Études Sci. 56 (1983), 387-410.

3. __ Hopf algebras of polynomial growth, J. Algebra 125 (1989), 408-417.

4. __ Elliptic Hopf algebras, J. London Math. Soc. (to appear).

5. $\frac{}{\text { tion). }}$. The Serre spectral sequence of a multiplicative fibration (in prepara- 
6. __ Torsion in loop space homology, preprint.

7. _ Dupin hypersurfaces are elliptic, preprint.

8. J. Friedlander and S. Halperin, An arithmetic characterisation of the rational homotopy groups of certain spaces, Invent Math. 53 (1979), 117-133.

9. T. H. Gulliksen, On the deviations of local ring, Math. Scand. 47 (1980), 5-20.

10. S. Halperin, Finiteness in the minimal models of Sullivan, Trans. Amer. Math. Soc. 230 (1977), 173-199.

11. __ Spaces whose rational homology and rational homotopy are both finite dimensional, Homotopie Algébrique et Algèbre Locale (J. M. Lemaire and J. C. Thomas, eds.) Astérisque 113/114 (1984), 198-207.

12. Universal enveloping algebras and loop space homology, preprint.

13. C. McGibbon and C. Wilkerson, Loop spaces of finite complexes at large primes, Proc. Amer. Math. Soc. 96 (1986), 698-702.

14. J. Milnor and J. C. Moore, On the structure of Hopf algebras, Ann. of Math. (2) 81 (1965), 211-264.

15. J. P. Serre, Homologie singulière des espaces fibrés, Ann. of Math. (2) 54 (1951), 425-505.

Institut de Mathématiques, Université Catholique de Louvain, B-1348 LouvAIN-LA-NeuVe, BelgiQue

Department of Mathematics, Scarborough College, University of Toronto, Scarborough, Canada M1C 1A4

U. F. R. DE MathématiQues, Université de Lille Flandre ARtois, F-59655 Villeneuve d'AscQ, Cedex, France 
